\section{$\underset{\text { hommes }}{\text { \& migrations }}$}

\section{Hommes \& migrations}

Revue française de référence sur les dynamiques

migratoires

$1317-1318 \mid 2017$

L'Europe en mouvement

\title{
Rap, antiracisme et identités locales en région liégeoise
}

\author{
Marco Martiniello
}

\section{OpenEdition \\ Journals}

\section{Édition électronique}

URL : http://journals.openedition.org/hommesmigrations/3917

DOI : 10.4000/hommesmigrations.3917

ISSN : 2262-3353

\section{Éditeur}

Musée national de l'histoire de l'immigration

\section{Édition imprimée}

Date de publication : 1 avril 2017

Pagination : 158-164

ISBN : 978-2-919040-38-4

ISSN : $1142-852 X$

\section{Référence électronique}

Marco Martiniello, «Rap, antiracisme et identités locales en région liégeoise », Hommes \& migrations [En ligne], 1317-1318 | 2017, mis en ligne le 01 avril 2017, consulté le 14 septembre 2020. URL : http:// journals.openedition.org/hommesmigrations/3917 


\section{RAP, ANTIRACISME ET IDENTITÉS LOCALES EN RÉGION LIÉGEOISE}

Par MARCO MARTINIELLO, Directeur de recherche FNRS,

Directeur du Centre d'études de l'ethnicité et des migrations (Cedem), université de Liège (Belgique).

Liège est la seconde plus grande ville francophone de Belgique avec environ 200 ooo habitants au cœur d'une zone urbaine de plus de 700 ooo personnes. Liège est la capitale économique de la Wallonie'. Après la révolution industrielle, Liège était devenue l'une des régions les plus riches et les plus développées au monde avec ses industries lourdes (charbon, acier, armes, verre). En raison de sa position géographique et de son économie florissante, Liège était une ville industrielle et industrieuse réputée jusqu'à son déclin à la fin 1960 et durant les années 1970. Aujourd'hui, la population est très multiethnique, multiraciale et multiculturelle avec de forts accents italiens. Les étrangers représentent environ $20 \%$ de la population de la ville. Plus de 100 nationalités sont représentées. Si nous incluons les générations suivantes des migrants, dont beaucoup sont maintenant des citoyens belges, la population d'origine étrangère s'élève certainement entre 25 et $30 \%$ de la population totale de la ville et de la proche région incluant des villes comme Seraing et Herstal ${ }^{2}$. La ville est clairement marquée par la super-diversité3.

Aujourd'hui, Liège constitue un exemple typique de ville industrielle du Nord de l'Europe déclinante, comme il en existe dans le Nord de la France, au Royaume-Uni ou en Allemagne. La production de charbon et de verre a totalement disparu. L'industrie de l'acier a perdu la plupart de ses emplois au cours des 25 dernières années. Le chômage n'a cessé de croître dans la même période pour atteindre $23 \%$ de la population apte au travail en $2013^{4}$. Malgré un système d'État providence et d'aide sociale relativement fort, la pauvreté est une réalité préoccupante dans la région. De plus, la petite criminalité urbaine liée à la consommation et au commerce des drogues est loin d'être négligeable. Politiquement, la région a une forte tradition socialiste et communiste, qui a probablement empêché la politisation du racisme comme dans d'autres villes belges. Mais le racisme quotidien et banal est une réalité sociale qui n'épargne pas la région. Les immigrés et leurs descendants sont souvent les boucs émissaires blâmés pour les maux de la ville. Ailleurs en Belgique, Liège est souvent pointée du doigt comme étant un endroit dangereux, sale rempli de chômeurs, de toxicomanes et de criminels.

Mais, au-delà ce sombre tableau, Liège est aussi une ville d'art et de culture ainsi qu'une ville de fête surnommée la « cité ardente » en raison 
de sa vie nocturne animée. L'université de Liège est un des plus grands employeurs de la région. Les finances de la ville ne permettent pas une politique cultuelle dispendieuse, mais, dans le système fédéral belge, la Fédération WallonieBruxelles est en charge des arts et de la culture et de nombreux projets et institutions sont parrainés à la fois dans les beaux-arts (l'opéra de Liège) et dans les arts plus populaires. La scène théâtrale est particulièrement dynamique comme du reste la scène hip-hop, dans laquelle de nombreux jeunes issus de l'immigration sont des acteurs clés.

\section{La musique, un porte-voix politique}

La musique comme mode d'expression et de participation socio-politique ${ }^{5}$ des populations mino- risées $^{6}$ à Liège sera dans les lignes qui suivent discutée à partir de deux cas issus de la culture hip-hop local en s'inspirant du modèle de Mark Mattern? : le collectif OHK (Organisation Hors du Kommun) et Ultras inferno 96. La recherche empirique qualitative a commencé en 2010 et une première phase a pris fin en 2014. Elle se poursuit à ce jour. Plusieurs outils de collecte de données ont été mis en œuvre. Tout d'abord, la littérature spécifique a été consultée. Deuxièmement, j'ai également recueilli les CD et les paroles des chansons. Troisièmement, j'ai pratiqué de l'observation lors d'événements musicaux. Outre la prise de notes écrites classique, j'ai fait des photos et, si possible, des enregistrements sonores et visuels pendant les événements musicaux. Quatrièmement, j'ai réalisé des entretiens semistructurés avec des artistes et des informateurs clés. Enfin, j'ai recueilli différentes données sur

5. Marco Martiniello, Jean-Michel Lafleur, "Ethnic minorities" cultural practices as forms of political expression: A review of the literature and a theoretical discussion on music ", in Journal of Ethnic and Migration Studies, vol. 34, $\mathrm{n}^{\circ}$ 8, 2008, pp. 11911215 ; Marco Martiniello, "Immigrants, ethnicized minorities and the arts: a relatively neglected research area ", in Ethnic and Racial Studies, vol. 38, n 8, 2015, pp. 1229-1235; William G. Roy, Reds, Whites, and Blues. Social Movements, Folk Music and Race in the United States, Princeton, Princeton University Press, 2010. 6. Jan Rath, Minorisering: De Sociale Constructie van Etnische Minderheden, Amsterdam, Sua, 1991. 7. Mark Mattern, Acting in Concert. Music, Community, and Political Action, New Brunswick, Rutgers University Press, 1998. 


\section{REPÉRAGE}

Internet, telles que le contenu des sites et des pages Facebook des artistes, des blogs et des forums. Youtube a été la source la plus précieuse de deux types de données : des clips vidéo musicaux et les commentaires qui apparaissent en dessous de ceux-ci. Ils constituent bien souvent un matériau riche à analyser. La recherche n'a pas mis l'accent au départ sur un style ou un genre musical précis. Toutefois, il est rapidement apparu que le rap constituait le style musical le plus important pour une bonne partie de la jeunesse de la région liégeoise, en particulier dans les quartiers populaires ouvriers qui sont aussi des quartiers historiques d'immigration.

\section{OHK, du rap de quartier à la politique locale}

OHK (Organisation Hors du Kommun) est un collectif de rap de La Préalle, un quartier ouvrier de la ville de Herstal en périphérie de Liège. Tout a commencé en 2006 par un atelier hip-hop lancé par une association locale subventionnée dans le cadre de la politique de la ville menée par le gouvernement fédéral belge. L'atelier était dirigé par un artiste local de rap et, comme c'est souvent le cas, il ne dura qu'un ou deux ans. Cependant, Illicite, un jeune Belgo-Italien, et Mujahid, un jeune Belgo-Turc, décidèrent de continuer l'expérience sous le nom de OHK, alternant jusqu'à ce jour les projets musicaux individuels et collectifs.

En 2010, Illicite poste un clip vidéo intitulé « Dans mon quartier "sur Youtube ${ }^{8}$. Tourné en noir et blanc avec très peu de moyens techniques, tout le clip se déroule sur un banc public du quartier sur lequel défilent des habitants de tous les âges et de toutes les origines, ainsi bien sûr qu'lllicite. Le clip présente une image plus ou moins lyrique, mais aussi brute et directe du quartier.
Illicite y raconte ce que signifie pour les habitants de vivre dans un tel endroit. D’un côté, il narre la misère urbaine et la pauvreté, les conditions de vie difficiles, l'économie irrégulière, un sentiment d'exclusion, la marginalisation et le malaise ressenti par les jeunes. Mais, d'un autre côté, le tableau dressé par Illicite est à bien des égards assez positif. Certes, la vie est difficile à La Préalle, mais Illicite aime vraiment son quartier. Il prononce l'expression "Dans mon quartier » plus de vingt fois. II fait l'éloge de la chaleur humaine, de la solidarité entre les habitants, et il célèbre son caractère multiculturel et les relations interculturelles harmonieuses, ainsi que l'absence de racisme. Comme le rappe Illicite, il veut «montrer tous ses côtés » de son quartier. II ne veut pas le quitter, mais le changer de l'intérieur. À travers le clip, Illicite raconte l'histoire et le quotidien de son quartier (fonction expressive de la musique). Au-delà, Illicite évoque un sens profond de la communauté locale ancré dans l'histoire de la classe ouvrière locale enrichie par l'arrivée et l'enracinement des travailleurs immigrés de nombreuses origines et de leurs familles. L'identité locale de La Préalle selon Illicite est multiculturelle. Ainsi, il écrit : "Dans mon quartier, y a tout le monde. Des cultures, des origines, des religions de tout le monde. Tout le monde connaît tout le monde9. " Ou encore : "Mon quartier c'est un bout d'Espagne, d'Italie, de Zaïre, de Maroc, de Kosovo, de Turquie. "

Mais c'est aussi clairement une identité de classe. "C'était le quartier des immigrés mineurs, des hommes qui allaient au charbon pour leur famille. " À travers ce morceau, Illicite propose clairement une identité locale ouvrière et multiculturelle source de fierté pour lui. Comme s'il fallait, dans cette période de fin de désindustrialisation, conserver la mémoire de ses racines ouvrières et immigrées pour se projeter dans 
l'avenir. La fonction délibérative de la musique est ainsi clairement exprimée.

La dimension protestataire n'est qu'indirectement présente dans cette chanson. Dans un passage, Illicite dit que son " quartier se bat chaque jour contre le système. Pour s'en sortir, il fabrique son propre système ». L'auteur s'en prend ici clairement aux pouvoirs économique et politique qui ont relégué le quartier dans la pauvreté et qui ont condamné ses habitants à développer des stratégies de survie par la débrouille dans l'économie informelle.

\section{De l'engagement citoyen à la campagne électorale}

Deux ans plus tard, les dimensions protestataire et pragmatique de sa musique apparaîtront de manière très évidente lorsqu'lllicite décidera de se présenter aux élections municipales de la ville de Herstal en octobre 2012 sous la bannière du Parti du travail de Belgique, un parti d'extrême gauche implanté dans la ville depuis des décennies. La campagne électorale d'Illicite consistera presqu'exclusivement en un clip vidéo posté sur Youtube le 25 septembre 2012 et intitulé « On vient changer la donne ${ }^{10} »$. Tourné en extérieur dans le quartier, mais cette fois en couleur afin de mettre en avant le rouge et le jaune - les couleurs traditionnelles de l'ultra gauche -, le clip se compose de deux parties. La première est rappée par Laurent, un candidat du PTB vêtu d'un T-shirt à l'effigie de Che Guevara. La seconde partie remet en scène Illicite, coiffé d'une casquette de base-ball et portant un foulard rouge et jaune de la section jeune du PTB. Dans les refrains, un groupe de jeunes du quartier de différentes origines reprend l'appel à la mobilisation lors des élections d'octobre 2012.

L’Enfant Pavé, membre du groupe Starflam et membre des Ultra Inferno. (c) Georges Kostenjak.

Le morceau s'inscrit incontestablement dans la veine des protest songs. Les deux rappeurs candidats aux élections mettent en cause le système politique local dominé depuis des décennies par le Parti socialiste qu'ils considèrent comme étant corrompu et clientéliste. Plus largement, ils s'en prennent à la droite et au système capitaliste dans son ensemble de manière assez classique. En un peu plus de 3 minutes et 30 secondes, ils passent en revue toute une série de thèmes comme le démantèlement du système public de santé, le chômage, la précarisation des emplois, la marginalisation des quartiers et de leur jeunesse, le sort réservé aux personnes âgées ou encore la stigmatisation des immigrés. Ils n'oublient pas une touche environnementaliste en insistant sur la pollution qui touche leur 


\section{REPÉRAGE}

ville et la région dans son ensemble. Par ailleurs, il est intéressant de remarquer qu'au-delà de la protestation ils avancent des propositions pour le développement de politiques publiques davantage en phase avec les besoins de la population. Plutôt qu'à la révolution, ils en appellent au changement par la participation électorale.

Le clip est un bon exemple d'action politique pragmatique par la musique. Son objectif évident est, en effet, d'obtenir le plus de voix possible lors des élections communales et, par conséquent, le plus d'élus possible au conseil municipal, en particulier pour la section jeunes du Parti du travail de Belgique, appelée Comac. Le PTB a au bout du compte obtenu $14 \%$ des suffrages lors des élections locales d'octobre 2012. Il est devenu le second parti de la ville derrière le Parti socialiste qui a conservé $51 \%$ des voix et donc le pouvoir à la mairie. Quatre candidats du PTB ont obtenu un siège de conseiller communal. Parmi ceux-ci, on retrouve un des jeunes que l'on aperçoit furtivement dans le clip de Laurent et d'Illicite, Maxime Liradelfo. Les deux rappeurs n'ont, quant à eux, pas passé la rampe. Bien évidemment, rien ne nous permet de prouver que le clip en question puisse expliquer tout ou partie de ce succès notable. Mais on peut faire l'hypothèse qu'il a joué un rôle dans le choix électoral d'une partie de la jeunesse locale.

\section{Ultras inferno 96 : « de l'énergie positive à l'aspect multiculturel »}

Outre ses institutions culturelles et son université, la ville de Liège possède aussi un club de football professionnel au passé européen glorieux qui milite aujourd'hui en première division belge. Le Standard de Liège reste aujourd'hui l'un des clubs les plus populaires du pays. Le stade de
Sclessin dans lequel il évolue est situé à la sortie de la ville, en bord de Meuse. Il est entouré d'usines et des restes d'un terril, vestige de l'époque minière. Cette saison, le club peut se vanter d'avoir 23000 abonnés et des clubs de supporters dans tout le pays, même en Belgique néerlandophone, au-delà de la frontière linguistique qui coupe la petite Belgique en deux.

Fondé notamment par les descendants d'immigrés italiens, les Ultras inferno 96 sont l'un de ces clubs de supporters, l'un des plus chauds, qui prend place en tribune T3 derrière le goal, tribune jadis appelée "pourtours terril ». Le charbonnage n'était, en effet, qu'à quelques centaines de mètres. Créés en 1996, les Ultras inferno se revendiquent, comme leur nom l'indique, du mouvement ultra. Selon les Ultras inferno, le " mouvement ultra " est né en Italie dans le courant des années 1960-1970". II s'inspire du " modèle anglais » tout en restant très différent de par son implication plus concrète dans le club ainsi que par ses diverses actions dans le stade pour encourager son équipe. Comme l'indique son nom, "ultra ", ce mouvement regroupe des supporters " acharnés » de leur équipe de football, prêts à les suivre partout, dans leur championnat comme en Europe, par tous les moyens possibles. Un ultra encourage son équipe par ses chants durant toute la rencontre, ses animations (« tifo ») à l'entrée des joueurs sur le terrain, etc. Un ultra peut aussi se distinguer par sa radicalité dans ses actions lorsque l'équipe joue mal : grève des encouragements, tribune bloquée, messages incendiaires, descentes aux entraînements... Un groupe ultra structuré peut avoir une influence énorme sur les décisions du club dans certains pays (politique de transfert, prix des tickets...).

En bref, un groupe ultra est un groupe constitué de fans invétérés faisant partie à part entière de la vie du club de football qu'ils supportent. 
Ils peuvent autant lui vouer un amour indéfini qu'une colère redoutable quand le groupe estime que le club "ne le respecte pas " à sa juste valeur ou se "fout " simplement des supporters en général. Certains estiment qu'il s'agit, en quelque sorte, du "syndicat " des fans. Cependant, chaque club, chaque groupe, chaque pays, chaque continent a ses spécificités, ses contextes qui font qu'il est impossible de définir un «profil type ".

Sur la page d'accueil du site web des Ultras inferno, on peut trouver cette carte de visite : " //s sont l'âme diabolique de Sclessin, ils défendent leur Club par vent et marée, ils protègent Liège comme le dernier bastion à conquérir, ils sont les ULTRAS-INFERNO, fraction antifasciste et antiraciste du Royal Standard Club de Liège. Rentrons avec eux dans l'univers des tribunes engagées et enragées ${ }^{12}$ !! » Ainsi, les ultras du Standard de Liège se définissent clairement comme antifascistes, antiracistes et de gauche. Ils font partie du réseau européen Alerta Network. Antifascist Movement $^{13}$ qui compte une vingtaine de groupes d'ultras de même obédience issus de différents clubs européens, et même un club israélien.

\section{Du stade au clip : savoir occuper une tribune}

À l'occasion de leur quinzième anniversaire en 2011, les Ultras inferno ont produit un clip de rap avec l'aide notamment de rappeurs très connus sur la scène hip-hop belge et européenne, dont Kaer et Pavé du groupe Starflam, un des groupes mythiques du hip-hop belge. Le clip, posté sur Youtube en 2011, dure plus de six minutes et emmène le spectateur au cœur du groupe et de ses valeurs sur un rythme très soutenu ${ }^{14}$. L'aspect visuel d'abord est très soigné. Le groupe a pris soin de mettre en scène des jeunes de toutes les origines, quelques femmes, dont la rappeuse Psychosa, et une personne handicapée. Che Guevara apparaît aussi sur une des banderoles déployées par le groupe dans le stade. Et le spectateur est invité à y prêter attention. Sanboy dit ainsi : "Si t’as pas capté la vibe, t’as qu'à regarder le tifo. " Le tifo qui présente justement l'effigie de Che Guevara.

La fonction expressive de ce clip, dédié à décrire la vie des Ultras inferno, est puissante. Ce cocktail savamment dosé dominé par la couleur rouge, qui est à la fois la couleur du club et une couleur politiquement très marquée, sert aussi de carte de visite. Ainsi, lorsque j'ai demandé aux ultras les paroles du clip, ils me les ont rapidement et aimablement envoyées, mais en anglais alors que le clip est principalement en français. Ce clip sert clairement à la représentation des Ultras inferno dans le mouvement ultra mondial. D'ailleurs, les commentaires, positifs ou négatifs, en provenance du monde entier ont été postés sur Youtube à son sujet.

Laspect protestataire du clip comporte néanmoins plusieurs dimensions. En premier lieu, des références sont faites aux interdits de stade pour des raisons sécuritaires. En les soutenant, car certains font partie des Ultras inferno, ils s'opposent clairement à l'appareil répressif d'État et en particulier à la police avec laquelle ils ont historiquement des rapports très tendus. En second lieu, l'opposition au fascisme et aux fascistes est affirmée avec force. Le groupe se montre prêt à aller jusqu'au combat physique contre eux. Sanboy dit que les Ultras inferno sont un «Noyau dur acharné qui importune tous les fachos». En troisième lieu, les ultras se positionnent contre le racisme. "Peu importe la couleur de peau ", dit le refrain. Enfin, le parti pris gauchiste traduit un rejet profond du système capitaliste même si 


\section{REPÉRAGE}

la politisation du groupe fait l'objet de bien des débats entre les ultras, lesquels sont gommés dans le clip.

L'aspect délibératif est primordial dans le clip. Les paroles font référence aux valeurs de fidélité, de loyauté, de courage, d'abnégation qui les caractérisent, tant dans la défense de leur équipe de football que de la ville et de la région de Liège. Plusieurs références sont faites à " ma " ville dans le clip. Par ailleurs, le clip fait l'éloge de la société multiculturelle et revendique les racines ouvrières des supporters du club. C'est le « repère des antifa, des prolétaires ». II n'est pas anodin que Pavé rappe deux phrases en néerlandais. Dans le contexte belge actuel marqué par de vives tensions entre les francophones et les néerlandophones, s'autoriser quelques phrases dans la langue de Vondel est une manière de rendre hommage non seulement aux nombreux supporters flamands du Standard, mais aussi à la Belgique multilingue. Remarquons en passant que Pavé se présentera aux élections communales à Liège en 2012 sous la bannière du PTB, un des seuls partis non divisé linguistiquement en Belgique. Il ne sera pas élu.

\section{Conclusion}

Les conclusions que l'on peut tirer de la brève présentation de ces deux cas dans un cadre général et théorique particulier sont nombreuses. En premier lieu, l'article montre qu'il est tout à fait pertinent d'étudier les " objets politiques non identifiés ${ }^{15}$ " tels que la musique dans une perspective relevant principalement de la socio- logie politique. C'est d'autant plus le cas à l'ère de la politique spectacle et des émotions virtuelles. II convient donc de redéfinir les frontières et les lieux de l'action politique qui ne se fait pas uniquement par le vote et dans les assemblées élues dans lesquelles officient des professionnels de la politique. La politique, peut aussi se faire par d'autres moyens moins conventionnels et dans d'autres lieux. C'est ce que cet article a voulu monter. En second lieu, l'article a fait émerger la nécessité de sortir d'une grille de lecture exclusivement ethnique ou culturelle au profit d'une approche plurielle et intersectionnelle, en passant notamment par la convocation du concept de classe sociale dans les débats sur les sociétés post-migratoires.

Enfin, cet article a aussi voulu relayer la parole d'une partie de la jeunesse d'un type de société en voie de disparition, la société urbaine industrielle du centre-nord européen. Certes, ni OHK, ni les Ultras inferno ne sont statistiquement représentatifs ni du hip-hop belge, ni de l'évolution du multiculturalisme, de l'antiracisme ou des nouvelles formes de mobilisation de l'ancienne classe ouvrière. Toutefois, ils représentent qualitativement un monde qui est en train de mourir et qui se bat pour survivre en utilisant notamment les canaux artistiques pour résister à la marche du néo-libéralisme qui les ignore sans être séduit par les sirènes du nationalisme et de l'extrême droite. N'est-ce pas aussi le rôle de la sociologie de faire voir ceux que personne ne voit ou ne veut plus voir et de montrer que, bien que certains résultats électoraux semblent dire le contraire, l'extrême droite est heureusement encore loin d'avoir colonisé tous les esprits? I 